\title{
Comparação biomecânica de CRIF e placa de reconstrução na estabilização de fraturas distais de fêmur em cães
}

\author{
[Biomechanical comparison of CRIF and reconstruction plate for stabilization of distal \\ femur fractures in dogs] \\ F. Paes ${ }^{1}$, C.R.A. Ferrigno ${ }^{2}$, P.V.T. Marinho ${ }^{3}$, C.A.M. Pereira ${ }^{4}$, T. Bregadioli ${ }^{1}$, \\ I. S. Dal-bó ${ }^{1}$, M.A. Ferraro ${ }^{1}$ \\ ${ }^{1}$ Aluno de pós-graduação - Universidade de São Paulo - São Paulo, SP \\ ${ }^{2}$ Universidade de São Paulo - São Paulo, SP \\ Ferrigno: \\ http://orcid.org/0000-0002-9805-989X \\ Marinho: \\ http://orcid.org/0000-0001-8399-0844 \\ Pereira: \\ http://orcid.org/0000-0002-9867-397X \\ Bregadioli: \\ http://orcid.org/0000-0002-2012-9800 \\ h. Dal-bó: \\ http://orcid.org/0000-0003-4652-8567 \\ Ferraro: \\ http://orcid.org/0000-0002-2949-6850 \\ ${ }^{3}$ Instituto Federal de Educação, Ciência e Tecnologia do Sul de Minas Gerais - Muzambinho, MG \\ ${ }^{4}$ Laboratório de Biomecânica do LIM-41/HC-FMUSP - São Paulo, SP
}

F. Paes:

http://orcid.org/0000-0001-5586-8083

\begin{abstract}
RESUMO
O presente estudo objetivou comparar, biomecanicamente, por meio do ensaio de compressão excêntrica, a resistência dos implantes Clamp and Rod Internal Fixation (CRIF) 5,0mm e placa de reconstrução $3,5 \mathrm{~mm}$ na fixação de fraturas distais de fêmur de cão. Foram utilizados 22 fêmures de 11 cadáveres de cão entre 2 e 7 anos de idade e peso corporal entre 20 e $40 \mathrm{~kg}$ e subdivididos em dois grupos, denominados grupo CRIF (GC - 11 fêmures) e grupo placa (GP - 11 fềmures).Para realização dos testes, foi simulada falha que mimetizasse uma fratura distal nos corpos de prova, por meio de uma osteotomia de até $0,5 \mathrm{~cm}$, realizada com auxílio de serra oscilatória, imediatamente proximal ao início da tróclea. Os implantes foram fixados segundo os padrões AOSIF, lateralmente ao fêmur, sendo utilizados três parafusos distais e cinco proximais ao foco de fratura. Foi empregado o programa de computador PMI para calcular o ponto máximo de resistência antes da falha e avaliaram-se as variáveis força máxima, deformação máxima real, rigidez, força intermediária e deformação intermediária real. Não foram encontradas diferenças estatisticamente significativas entre os GC e GP quanto às variáveis avaliadas, sugerindo que ambos os implantes são boas opções de fixações para tais fraturas.
\end{abstract}

Palavras-chave: cães, fraturas femorais, fixadores internos, placas ósseas

\begin{abstract}
This study aims to compare the biomechanical properties, through compression eccentric test, of the resistance of the 5.0mm Clamp and Rod Internal Fixation Implants (CRIF) and 3.5mm reconstruction plate in fixing dog femur distal fracture. It was used 22 femurs from 11 dog cadavers with age between 2 and 7 years old and body weight between 20 and 40kg, subdivided into two groups, called CRIF Group (CG - 11 femurs) and plate group (PG - 11 femurs). A distal fracture in the specimens with a gap of $0.5 \mathrm{~cm}$ osteotomy, was simulated to perform the test, performed by the oscillating saw, just proximal to the beginning of the trochlea. The implants were fixed by the standards AO / SIF, laterally to the femur, using three distal screws and five proximal to the fracture site. PMI computer program was used to calculate the maximum point of resistance before failure and evaluated the variables maximum strength, real maximum deformation, stiffness, intermediate strength and real intermediate deformation. No statistically significant differences were found between the GC and GP as the variables evaluated, suggesting that both implants are good fixation options for such fractures.
\end{abstract}

Keywords: dogs, femoral fractures, internal fixators, bone plates, biomechanical

Recebido em 3 de outubro de 2016

Aceito em 14 de julho de 2017

E-mail: fpaesmv@gmail.com 


\section{INTRODUÇÃO}

As fraturas do segmento distal, em cães, representam de 20 a $25 \%$ das fraturas femorais e $11 \%$ de todas as fraturas diafisárias (Unger et al., 1990; Braden et al., 1995; Piermattei et al., 2009). As fraturas envolvendo as epífises distais femorais são relativamente comuns em animais jovens com idade entre quatro e 11 meses, sendo as fraturas de Salter tipo I e II vistas com mais frequência (Brinker, 1974; Grauer et al., 1981; Olmstead, 1984; Engel; Kneiss, 2014). As fraturas supracondilares são observadas mais comumente em animais adultos. Ambos os tipos de fraturas apresentam desafios biomecânicos similares relativos à redução e à fixação (Unger et al., 1990; Braden et al., 1995).

As fraturas distais de fêmur requerem intervenção cirúrgica precoce e a manipulação delicada dos tecidos moles associada à redução anatômica adequada ao tipo de fratura, assim como o alinhamento rotacional e angular. A máxima estabilidade é fundamental para o sucesso do tratamento, o que facilita o suporte de peso e evita rigidez articular, complicações relacionadas à união óssea e doenças articulares associadas à fratura (Tomlinson, 2005).

Na grande maioria dos casos, essas fraturas têm sido tradicionalmente tratadas por meio de pinos cruzados, pinos de Rush, placas de compressão dinâmica, placas de reconstrução e fixadores externos (Piermattei et al., 2009). No entanto, têm-se observado algumas desvantagens na utilização desses implantes, tanto em relação à estabilidade, à modelagem dos implantes, quanto em relação ao suprimento sanguíneo, já que nenhuma delas permite uma associação adequada dessas características, sendo a placa de reconstrução a que mais se enquadra nesses prérequisitos, pois permite estabilidade adequada e modelagem (Tomlinson, 2005).

O desenvolvimento de implantes segue a filosofia contemporânea de manter o suprimento sanguíneo intacto, reduzindo a área de contato osso-implante, bem como a estabilidade adequada de acordo com o tipo de fratura (Jain et al., 1999; Zahn et al., 2008). O sistema Clamp and Rod Internal Fixation (CRIF) foi desenvolvido para o uso veterinário, favorecendo alguns conceitos de osteossíntese biológica (Haerdi-Landerer et al., 2002), e tem mostrado versatilidade na aplicação clínica, principalmente em fraturas em que um dos fragmentos, proximal ou distal, é pequeno o suficiente para a aplicação de determinados implantes (Zahn; Matis, 2004; Zahn et al., 2008).

São escassos os trabalhos na literatura revisada que avaliem o uso do CRIF e da placa de reconstrução nas fraturas distais de fêmur de cães (Florin et al., 2005; Higgins et al., 2007; Bonin et al., 2014). Em razão disso, este estudo teve por objetivo comparar, biomecanicamente, a fixação das fraturas distais de fêmur de cães com CRIF e placa de reconstrução, com a hipótese de que há diferença biomecânica quanto à resistência de ambos os implantes para correção desse tipo de fratura

\section{MATERIAL E MÉTODOS}

Foram coletados e utilizados 11 pares de fêmures de cão, totalizando 22 fêmures, provenientes do Serviço de Patologia, sob aprovação da Comissão de Ética no Uso de Animais, com protocolo $\mathrm{n}^{\mathrm{o}}$ 4607210314. Todos os animais vieram a óbito por motivos não relacionados a este estudo, sendo incluídos cadáveres de cães com idade entre dois e sete anos, peso entre 20 e $40 \mathrm{~kg}$, não havendo predileção por raça ou sexo, sem histórico ou diagnóstico de doença nutricional, óssea ou degenerativa, fratura em colo femoral e histórico de artroplastia. Além disso, foram excluídos quaisquer espécimes que apresentassem sinais de osteoporose que, durante a fixação dos implantes, demonstrasse pobre fixação dos parafusos.

As amostras foram coletadas logo após a morte dos animais, sendo inicialmente removidas a pele e a musculatura remanescente (esqueletização), sendo no fêmur proximal removido por desarticulação coxofemoral, e o distal pela desarticulação fêmoro tíbio patelar. Em seguida, foram armazenados em freezer convencional, com temperatura de $-20^{\circ} \mathrm{C}$, quando da impossibilidade de realizar o ensaio no mesmo dia. As peças foram armazenadas em embalagens plásticas com dimensões de $50 \mathrm{~cm}$ de altura e $30 \mathrm{~cm}$ de largura. Em cada embalagem, foi acondicionado um par de fêmures em sacos plásticos idênticos transparentes com identificação da idade, sexo, raça, peso do cão proveniente feita com caneta permanente resistente a água. 
Com auxílio de serra oscilatória, foi realizada uma falha de $0,5 \mathrm{~cm}$ em todos os fêmures, imediatamente proximal ao início da tróclea. Para correta localização da falha, padronizou-se com um paquímetro para que a distância entre os côndilos femorais para ambos os fêmures (Fig. 1) fosse equivalente à distância desde a falha até $o$ limite distal do fêmur, medido em centímetros, a fim de testar os implantes sob as mesmas condições de testes de compressão e flexão.

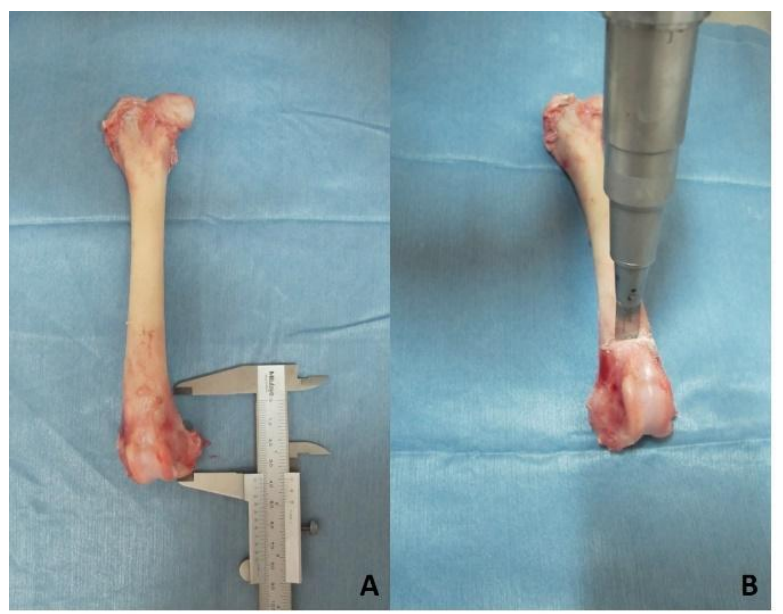

Figura 1. Mensuração com paquímetro da distância em $\mathrm{cm}$ a se mimetizar fratura distal de fêmur de cadáver de cão (A); Criação da falha por meio de serra oscilatória (B).

Para realização dos testes, foi adaptado um dispositivo cilíndrico de polimetilmetacrilato (PMMA) para mimetizar a força do acetábulo exercida na cabeça do fêmur no eixo axial. Os corpos de prova foram posicionados longitudinalmente e, para evitar alterações na descarga de força, uma vez que há diferentes tamanhos de cabeça femoral em apenas um tamanho de dispositivo semelhante ao acetábulo, foi utilizado PMMA, para moldar o formato da cabeça femoral ao dispositivo. Da mesma forma, foi utilizado um dispositivo, em formato de copo para acoplar a porção distal do fêmur, sendo adaptado também o uso de PMMA para evitar o deslizamento do côndilo femoral (Fig. 2).

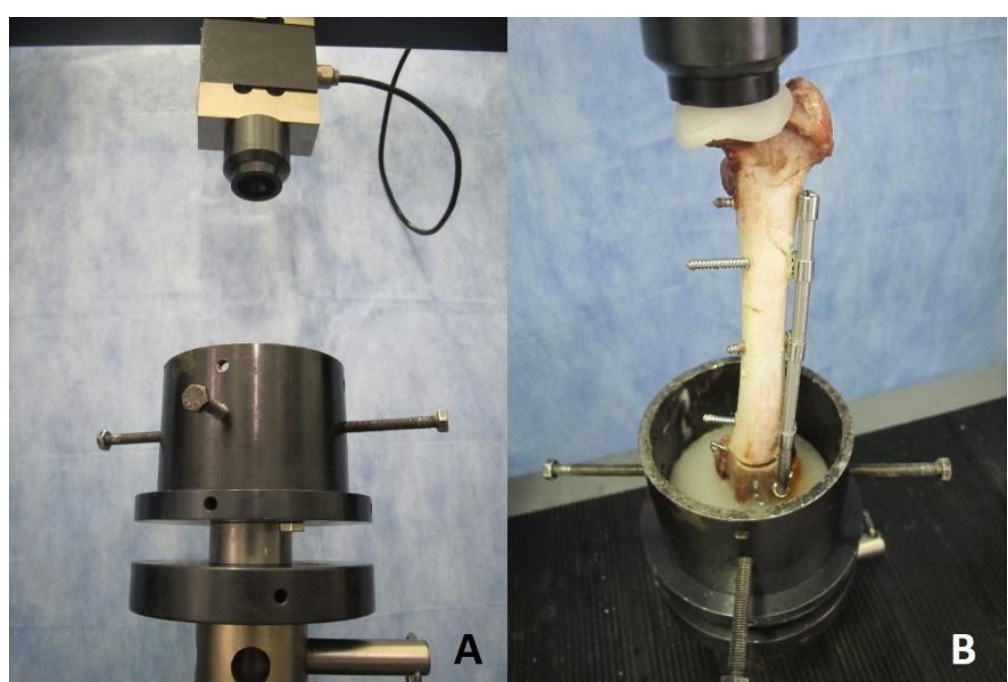

Figura 2. Dispositivo para realização dos ensaios de compressão e flexão (A) Adaptação para cabeça e côndilos femorais de cadáveres de cão, no dispositivo pronto para ensaio. 
Este estudo foi realizado de forma prospectiva, comparativa e aleatória. Os animais incluídos na pesquisa foram distribuídos aleatoriamente, em dois grupos, por meio da realização de sorteio, que determinou o implante a ser utilizado para a fixação (CRIF ou placa de reconstrução) e qual lado seria submetido ao tratamento específico. Cada lado do par pertencia a um grupo:

- $\quad$ grupo CRIF (GC)

- $\quad$ grupo placa de reconstrução (GP)

Ambos os membros foram colocados em um mini torno/morsa de bancada para facilitar a redução das fraturas para fixação dos implantes, respeitando um mesmo padrão de posicionamento dos parafusos para ambos os grupos, ou seja, mantendo pelo menos três parafusos distais (seis corticais) e cinco parafusos proximais (10 corticais). Para o grupo CRIF, foram utilizados CRIFs (Clamp and Rod Internal Fixation Depuy Synthes VET - Johnson \& Johnson - West Chester $\square$ Pensilvânia) médios com barra de cinco milímetros, assim como standard e end clamps médios, e para o grupo placa, utilizada placa de reconstrução 3,5mm (Placa de reconstrução pélvica $\square$ Ortosíntese $\quad-$ São Paulo $\quad-$ Brasil) (Fig. 3).

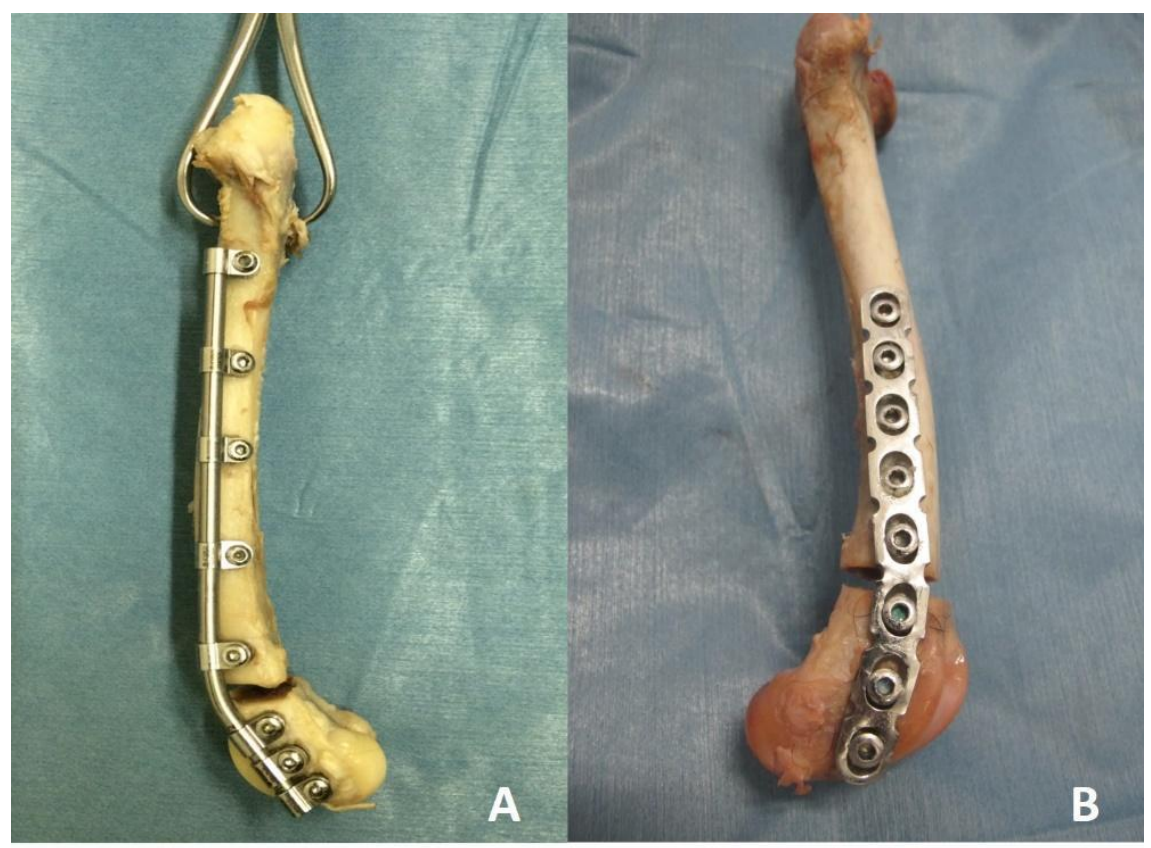

Figura 3. Padrão de posicionamento dos parafusos no grupo CRIF (A) e no grupo placa (B) em fêmur de cadáver de cão.

A comparação da resistência dos implantes dos grupos GC e GP foi realizada por meio de ensaio de flexão e compressão na máquina de ensaios universal, utilizando célula de carga de $3000 \mathrm{kgf}$ (quilograma-força) e velocidade de deslocamento de $5 \mathrm{~mm} / \mathrm{min}$. Foram estabelecidos como padrão de segurança valor de carga máxima de $1500 \mathrm{kgf}$, deslocamento máximo de $25 \mathrm{~mm}$ e término de ensaio, caso a carga de ruptura ultrapasasse $80 \%$ da carga máxima.

O parâmetro força foi enviado ao computador Apple com auxílio de um sistema de aquisição analógica TRACOMP-W95 (TRCV61285), permitindo a gravação dos parâmetros e dos gráficos para posterior análise.

Os resultados dos grupos placa (GP) e grupo CRIF (GC) foram discriminados pelas variáveis média, mediana, desvio-padrão, mínimo e máximo, das variáveis força máxima $(\mathrm{N})$, deformação máxima real $(\mathrm{mm})$, rigidez 1 $(\mathrm{kgf} / \mathrm{mm})$, rigidez 2 (kgf/mm), força intermediária $(\mathrm{N})$ e deformação intermediária real (mm). 
Foram realizados 22 ensaios de 11 pares. Foi realizada análise estatística para os dados de força máxima, rigidez 1 e rigidez 2 que apresentaram distribuição normal (utilizado o teste de normalidade de Anderson-Darling) e igualdade de variâncias, foi utilizado o t-pareado. Para os dados deformação máxima, força intermediária e deformação intermediária que não apresentaram distribuição normal ou igualdade de variâncias, foi utilizado o teste de Wilcoxon.

Todos os dados obtidos foram submetidos ao teste de normalidade de Anderson-Darling e ao teste de Igualdade de Variâncias, a fim de se verificar se os valores apresentavam homocedasticidade, para então decidir qual o teste estatístico seria utilizado.

Para os dados de força máxima, rigidez 1 e rigidez 2 que apresentaram distribuição normal e variâncias iguais, foi utilizado o teste t pareado, a fim de se identificarem possíveis diferenças entre os tratamentos.

Para os dados referentes à deformação máxima, força intermediária e deformação intermediária que não apresentaram distribuição normal e/ou não apresentaram variâncias iguais, foi utilizado o teste de Wilcoxon para se identificarem possíveis diferenças entre os tratamentos.

O grau de significância estabelecido para os testes estatísticos foi de $5 \%(\mathrm{P}<0,05)$. Os testes estatísticos foram realizados em programa de computador (Minitab Statistical Software, versão 17, 2015).

\section{RESULTADOS}

Foram descritas as variáveis pela média, pela mediana e pelo desvio-padrão, mínimo e máximo, e comparados o grupo $\operatorname{CRIF}(\mathrm{GC})$ e o grupo placa (GP) (Tab. 1).

Foi possível observar valores maiores no GP do que no $\mathrm{GC}$, das variáveis testadas, com exceção dos valores de deformação. Ou seja, valor de força máxima menor para o GC $(2.198,55 \pm 1.006,39 \mathrm{~N})$ quando comparado ao GP $(2518,72 \pm 672,02)$, entretanto sem diferença significativa $(\mathrm{P}=0,209)$ e quanto a força intermediária valores do GP maiores $(757,15 \pm 391,56)$ quando comparados ao GC $(608,00 \pm 328,23)$, porém da mesma forma sem diferença significativa $(\mathrm{P}=0,2402)$ (Fig. 4). Quando comparados os grupos nos valores referentes a rigidez 1, foi observado valores também maiores para o GP $(375,60 \pm 173,74)$ quando comparado ao GC $(287,40 \pm 199,52)$, porém sem diferença significativa $(\mathrm{P}=0,267)$, assim como em rigidez 2 , os valores para o GP também foram maiores $(699,29 \pm 238,18)$ e para o GC $(578,75 \pm 413,86)$, também sem diferença significativa $(\mathrm{P}=0,434) \quad$ (Fig. 5). Quando comparados os valores de deformação máxima real foi observado valores maiores para o GC $(9,67 \pm 4,18)$ do que para o GP $(6,96 \pm 2,99)$, porém também sem diferença significa $(\mathrm{P}=$ 0,123), assim como quando comparados os valores de deformação intermediária real do $\mathrm{GC}$ $(4,00 \pm 2,85)$ em relação ao GP $(2,87 \pm 1,03)$ também sem diferença significativa $(\mathrm{P}=0,4131)$ (Fig. 6).

Tabela 1. Valores de média, mediana, desvio padrão, mínimo e máximo para comparação de resistência entre os grupos CRIF e Placa

\begin{tabular}{ccccccccccc}
\hline & \multicolumn{1}{c}{ Grupo CRIF } & \multicolumn{4}{c}{ Grupo placa } \\
\hline Variáveis & Média & Mediana & $\begin{array}{c}\text { Desvio- } \\
\text { padrão }\end{array}$ & Mínimo & Máximo & Média & Mediana & $\begin{array}{c}\text { Desvio- } \\
\text { padrão }\end{array}$ & Mínimo & Máximo \\
\hline $\begin{array}{c}\text { Força máxima (N) } \\
\text { Deformação máxima } \\
\text { real (mm) }\end{array}$ & 2198,55 & 2347,04 & 1006,39 & 801,97 & 3641,96 & 2518,72 & 2523,62 & 672,02 & 1302,28 & 3538,96 \\
$\begin{array}{c}\text { Rigidez 1 (N/mm) } \\
\text { Rigidez 2 (N/mm) }\end{array}$ & 28,67 & 8,42 & 4,18 & 4,04 & 19,53 & 6,96 & 6,44 & 2,99 & 2,94 & 12,53 \\
$\begin{array}{c}\text { Força intermediária } \\
\text { (N) }\end{array}$ & 608,00 & 426,74 & 328,23 & 294,30 & 1236,06 & 757,15 & 640,10 & 391,56 & 198,65 & 1508,29 \\
$\begin{array}{c}\text { Deformação } \\
\text { intermediária real } \\
\text { (mm) }\end{array}$ & 4,00 & 3,35 & 2,85 & 0,81 & 11,06 & 2,87 & 2,78 & 1,03 & 0,84 & 4,28 \\
\hline
\end{tabular}

Fonte: (Paes, F. 2015). 


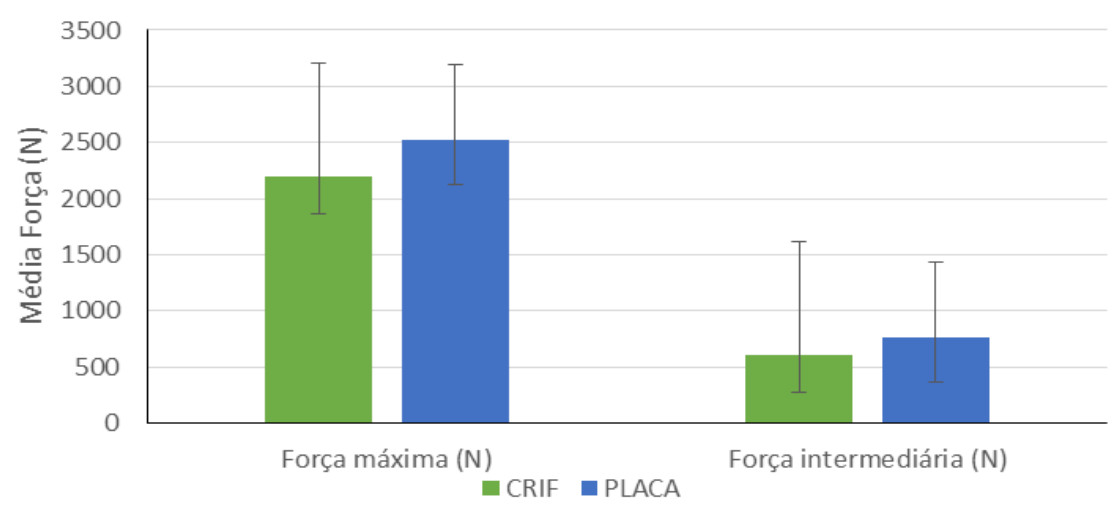

Fonte: (Paes, F., 2016).

Figura 4. Valores de média das variáveis força máxima $(\mathrm{N})$ e força intermediária $(\mathrm{N})$ dos grupos placa e CRIF com desvio padrão.

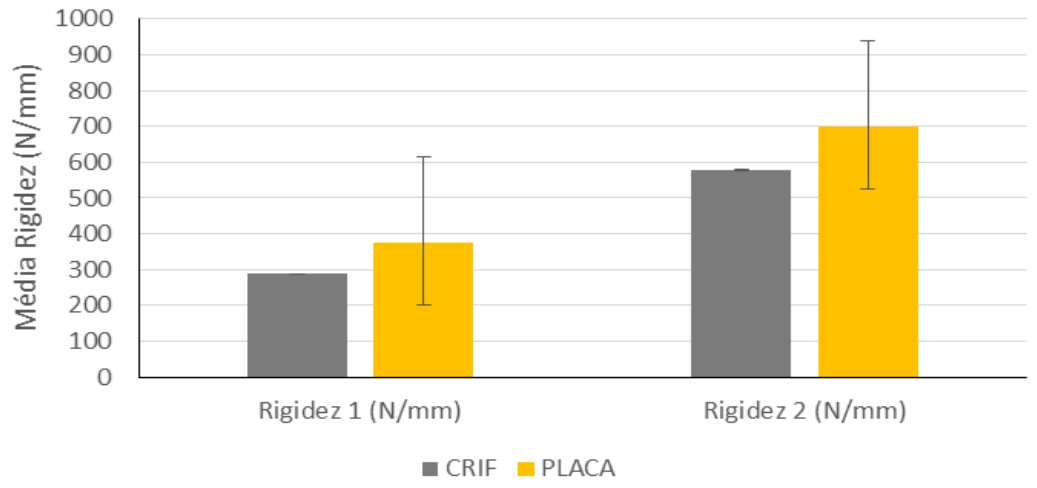

Fonte: (Paes, F., 2016).

Figura 5. Valores de média das variáveis rigidez $1(\mathrm{~N} / \mathrm{mm})$ e rigidez $2(\mathrm{~N} / \mathrm{mm})$ dos grupos placa e CRIF com desvio padrão.

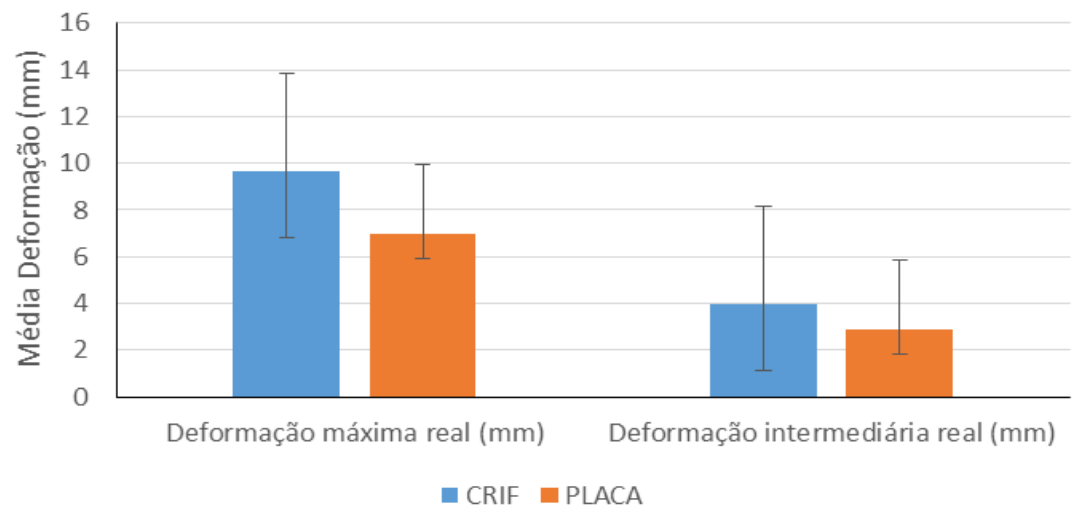

Fonte: (Paes, F., 2016).

Figura 6. Valores de média das variáveis deformação máxima real $(\mathrm{mm})$ e deformação intermediária real (mm) dos grupos placa e CRIF com desvio padrão. 
Um ensaio foi excluído da análise estatística por apresentar disparidade total do tempo e características de avaliação pela Kratos, por se tratar de um animal cuja a idade foi imprecisa, decidiu-se por caracterizar tais dados como valores outliers, discriminados nos gráficos que serão apresentados.

\section{DISCUSSÃO}

A hipótese de que haveria uma diferença significativa quanto à resistência do CRIF $5 \mathrm{~mm}$ em relação às placas de reconstrução $3,5 \mathrm{~mm}$ na fixação de fraturas distais de fêmur, não pôde ser completamente confirmada. Durante a realização dos testes, foi possível determinar resistência ao arqueamento do CRIF muito semelhante à conferida pelas placas, porém com valores de deformação maiores, supostamente por alguma fragilidade do CRIF anteriormente descrita apenas às forças de torsão (Zahn et al., 2008).

Um modelo com gap foi escolhido em concordância com estudos prévios de Silbernagel et al.(2004), uma vez que a maioria das fraturas em ossos longos de pequenos animais é cominutiva sem suporte ósseo (Braden et al., 1995; Johnson et al., 1998; Reems et al, 2003). Assim, o modelo com gap representa o pior cenário dentro da representação de modelos de fraturas de ossos longos. Além disso, sabe-se que cargas adicionais em modelos sem gap ou com oclusão de gap ocorrem pelo contato ósseo, o que pode levar à falha precoce do modelo durante o ensaio biomecânico (Johnston et al., 1991; Miclau et al., 1995; Silbernagel et al., 2004), tendo ocorrido fraturas dos ossos ou modelos de ossos sem gap, próximo aos orifícios dos parafusos, e não por deformação do implante (Miclau et al., 1995).

Independentemente das propriedades biomecânicas do CRIF e de a placa de reconstrução serem previamente descritas como diferentes, há a hipótese de que tiveram atividades biomecânicas semelhantes pela disposição e pelo tamanho dos parafusos utilizados para mimetizar as fraturas durante os ensaios (Zahn et al., 2008).

O comprimento de uma placa, assim como a homogeneidade de distribuição dos parafusos, influencia diretamente o suporte de carga da placa e dos próprios parafusos, além do suporte às forças de compressão. Define-se ainda que o comprimento e a distribuição ideal de parafusos contemplem a característica e a função exercida pela placa, além da extensão da placa perante as dimensões do foco de fratura ser maior que dois terços do foco de uma fratura cominutiva e maior em oito a 10 vezes quando em uma fratura transversa (Gautier; Sommer, 2003; Stoffel et al., 2003). Com base nisso, no presente estudo, estabeleceu-se que o tamanho, a disposição e o número de parafusos seriam exatamente o mesmo em ambos os grupos testados, sendo padronizados para tamanho dos espécimes coletados como adequado para as placas de reconstrução 3,5mm e CRIF 5mm, assim como o posicionamento de ambos os implantes no osso e o retorcimento deles realizados de maneira semelhante, a fim de limitar qualquer diferença entre os ensaios.

A escolha de ensaio de compressão com carga axial segue o modelo descrito por outros autores de criar um momento de arqueamento devido ao gap criado e à fixação abaxial dos implantes, que se torna susceptível à ação das cargas e arqueamento do implante (Olmstead, 1984; Zahn et al., 2008). As forças de tensão e arqueamento representam mais de $90 \%$ das forças fisiológicas exercidas durante o apoio do membro (Perren $e t$ al., 1988) e, por isso, neste estudo foi realizado ensaio excêntrico que gera forças de compressão e flexão e testa, portanto, resistência ao arqueamento.

Baseando-se nas propriedades estruturais, previase que a barra de conexão de $5 \mathrm{~mm}$ do CRIF, por ser cilíndrica, resistiria mais às cargas se comparada a uma placa de reconstrução prismática quando submetida ao teste de compressão e, consequentemente, à força de arqueamento. A área de momento de inércia, que é uma propriedade de formas utilizadas para prever o stress e deformações, para o CRIF $\left(30,66 \mathrm{~mm}^{4}\right)$ já foi anteriormente provada como maior do que para as placas, nesse caso especificamente as LC-DCP $\left(18,22 \mathrm{~mm}^{4}\right.$ entre os orifícios das placas, $13,4 \mathrm{~mm}^{4}$ através dos orifícios das placas) (Bonin et al., 2014), porém foram calculados, para a placa de reconstrução utilizada nos ensaios, valores com a placa em eixo horizontal inferiores aos do CRIF $\left(16,05 \mathrm{~mm}^{4}\right.$ entre os orifícios da placa, 11 , $26 \mathrm{~mm}^{4}$ nos orifícios da placa). Entretanto, se for 
avaliada a placa no eixo vertical, encontram-se valores superiores ao CRIF $\left(89,48 \mathrm{~mm}^{4}\right.$ entre os orifícios da placa, $215,44 \mathrm{~mm}^{4}$ nos orifícios da placa), e, muito provavelmente por esse motivo, a placa de reconstrução nesse ensaio de compressão, que também sofreu influência de flexão, tenha tido cargas exercidas em planos cuja a área de momento de inércia possa ter variado entre seu menor e maior valor e, por isso, tenha resultado em maiores valores de rigidez $\mathrm{e}$ menores valores de deformação quando comparada ao grupo placa, mesmo que sem diferença estatística. Isso demonstra ainda a sensibilidade da placa de reconstrução ao alinhamento em relação à aplicação da carga, diferentemente do CRIF em que a aplicação de carga numa barra cilíndrica independe do plano, já que o momento de área de inércia é o mesmo (Zahn et al., 2008).

Zahn et al. (2008) já haviam reportado, previamente, que o CRIF com diâmetro $5 \mathrm{~mm}$ teve valores de cargas e resistência ao arqueamento significativamente superiores que ambas as placas DCP e LCDCP $3.5 \mathrm{~mm}$, entretanto, tal análise foi realizada apenas em modelos de substitutos de osso Canevasit com gap sob forças de compressão e torsão em uma carga única até a falha do implante. Assim também, Bonin et al. (2014) compararam as propriedades biomecânicas da placa LC-DCP $3,5 \mathrm{~mm}$ e CRIF $5 \mathrm{~mm}$, associados ao pino intramedular em fêmures de cadáver de cão, e, contrariamente a Zahn et al. (2008), não encontraram diferença significativa na resistência ao arqueamento, nos valores de carga ou no ângulo de arqueamento até a falha do implante para os grupos de tratamento LC-DCP/pino e CRIF/pino.

Contrariamente aos mesmos estudos de Zahn et al. (2008) e similarmente ao estudo de Bonin et al. (2014), não se encontrou diferença significativa em ambos os grupos de tratamento para deformação máxima e intermediária. Entretanto, sabe-se que, como as placas de reconstrução são desenhadas para serem mais aptas a contornar o limite ósseo, consequentemente apresentam rigidez de deformação de arqueamento mais baixa (Robertson et al., 2009) para força máxima e intermediária e para rigidez, neste trabalho, denominada como rigidez 1 e 2 . Além disso, denota-se a importância da metodologia deste projeto, uma vez que, estabelecendo a avaliação pré-contato do gap (rigidez 1) e pós-contato do gap (rigidez 2), foi possível avaliar isoladamente os efeitos das forças de arqueamento diretamente sobre o implante e não sobre o tecido ósseo.

Define-se como limitações a este estudo o tipo de testes utilizados, uma vez que, mimetizada a força de compressão assim como já testada anteriormente (Goh et al., 1989) em testes compressivos que simulam o aumento da carga pós-operatória em fêmur, ainda assim não se avaliaram outras forças sabidamente essenciais a movimentos fisiológicos do cão (tração, torção), que poderiam talvez demonstrar diferenças significativas, assim como já citado em trabalhos anteriormente referenciados, a fim de se comparar o CRIF a outros implantes em que este demonstrou, por exemplo, maior fragilidade a outras forças como torção.

\section{CONCLUSÃO}

Conclui-se que ambos os implantes demonstram resistência e rigidez semelhantes, o que sugere que a utilização do CRIF possa ser mais uma possível opção a tais fixações.

\section{REFERÊNCIAS}

BONIN, G.A.; BAKER, S.T.; DAVIS, C.A. et al. In vitro biomechanical comparison of $3.5 \mathrm{~mm}$ LCDCP/intramedullary rod and $5 \mathrm{~mm}$ clamp-rod internal fixator (CRIF)/intramedullary rod fixation in a canine femoral gap model. Vet. Surg., v.43, p.860-868, 2014. BRADEN, T.D.; EICKER, S.W.; ABDINOOR, D.; PRIEUR, W.D. Statistical report characteristics of 1000 femur fractures in the dog and cat. Vet. Comp. Orthop. Traumatol., v.8, p.203-209, 1995.

BRINKER, W.O. Fractures. In: Canine Surgery Canine surgery. 2.ed. Santa Barbara: American Veterinary Publications, 1974. p.949-1048.

ENGEL, E.; KNEISS, S. Salter-harris fractures in dogs and cats considering problems in radiological reports--a retrospective analysis of 245 cases between 1997 and 2012. Berl. Munch. Tierarztl. Wochenschr., v.127, p.77-83, 2014

FLORIN, M.; ARZDORF, M.; LINKE, B.; AUER, J.A. Assessment of stiffness and strength of 4 different implants long-bone fracture model using a bone substitute. Vet. Surg., v.34, p.231-238, 2005.

GAUTIER, E.; SOMMER, C. Guidelines for the clinical application of the LCP. Injury, v.34, Suppl.2, p.63-76, 2003. 
GOH, J.C.; ANG, E.J.; BOSE, K. Effect of preservation medium on the mechanical properties of cat bones. Acta Orthop. Scand., v.60, p.465-467, 1989.

GRAUER, G.F.; BANKS, W.J.; ELLISON, G.W. Incidence and mechanisms of distal femoral physeal fractures in the dog and cat. J. Am. Anim. Hosp. Assoc., v.17, p.579-586, 1981.

HAERDI-LANDERER, C.; STEINER, A.; LINKE, B. et al. Comparison of double dynamic compression plating versus two configurations of an internal veterinary fixation device: results of in vitro mechanical testing using a bone substitute. Vet. Surg., v.31, p.582-588, 2002.

HIGGINS, T.F.; PITTMAN, G.; HINES, J.; BACHUS, K.N. Biomechanical analysis of distal femur fracture fixation: fixed-angle screw-plate construct versus condylar blade plate. J. Orthop. Trauma, v.21, p.43-46, 2007.

JAIN, R.; PODWORNY, N.; HUPEL, T.M. et al. Influence of plate design on cortical bone perfusion and fracture healing in canine segmental tibial fractures. J. Orthop. Trauma, v.13, p.178-186, 1999.

JOHNSON, A.L.; SMITH, C.W.; SCHAEFFER, D.J. Fragment reconstruction and bone plate fixation versus bridging plate fixation for treating highly comminuted femoral fractures in dogs: 35 cases (1987-1997). J. Am. Vet. Med. Assoc., v.5, p.11571161, 1998.

JOHNSTON, S.A.; LANCASTER, R.L.; HUBBARD, R.P.; PROBST, C.W.A Biomechanical comparison of 7-hole $3.5 \mathrm{~mm}$ broad and 5-hole $4.5 \mathrm{~mm}$ narrow dynamic compression plates. Vet. Surg., v.20, p.235239, 1991.

MIClAU, T.; REMIGER, A.; TEPIC, S. et al. Mechanical comparison of the dynamic compression plate, limited contact-dynamic compression plate, and point contact fixator. J. Orthop. Trauma, v.9, p.17-22, 1995.

OLMSTEAD, M. Fractures of the femur. In: BRINKER, W.; HOHN, R.; PRIEUR, W. (Eds.). Manual of internal fixation in the small animals. New York: Springer-Verlag, 1984. p.165-175.

PERREN, S.M.; CORDEY, J.; RAHN, B.A. et al. Early temporary porosis of bone induced by internal fixation implants. Clin. Orthop. Related Res., v.232, p.139-151, 1988
PIERMATTEI, D.L.; FLO, G.L.; DECAMP, C.E. Fratura do fêmur e da patela. In: Ortopedia $e$ tratamento de fraturas de pequenos animais. 4.ed. São Paulo: Manole, 2009. p.580-636.

REEMS, M.R.; BEALE, B.S.; HULSE, D.A. Use of a plate-rod construct and principles of biological osteosynthesis for repair of diaphyseal fractures in dogs and cats: 47 cases (1994-2001). J. Am. Vet. Med. Assoc., v.223, p.330-335, 2003.

ROBERTSON, C.; CELESTRE, P.; MAHAR, A.; SCHWARTZ, A. Reconstruction plates for stabilization of mid-shaft clavicle fractures: differences between nonlocked and locked plates in two different positions. J. Shoulder Elbow Surg., v.18, p.204-209, 2009.

SILBERNAGEL, J.T.; JOHNSON, A.L.; PIJANOWSKI, G.J.; EHRHART, N.; SCHAEFFER, D.J.A mechanical comparison of $4.5 \mathrm{~mm}$ narrow and $3.5 \mathrm{~mm}$ broad plating systems for stabilization of gapped fracture models. Vet. Surg., v.33, p.173-179, 2004.

STOFFEL, K.; DIETER, U.; STACHOWIAK, G. et al. Biomechanical testing of the LCP - How can stability in locked internal fixators be controlled? Injury, v.34, Suppl.2, p.SB11-SB19, 2003.

TOMLINSON, J. Fractures of the distal femur. In: JOHNSON, A.L.R.; HOULTON, J.E.F.; VANNINI, R. (Eds.). AO principles of fracture management in the dog and cat. Davos Platz, Switzerland: Thieme, 2005. p.297-303.

UNGER, M.; MONTAVON, P.; HEIM, U. Classification of fractures of long bones in the dog and cat introduction and clinical application. Vet. Comp. Orthop. Traumatol., v.3, p.41-50, 1990.

ZAHN, K.; FREI, R.; WUNDERLE, D. et al. Mechanical properties of 18 different AO bone plates and the clamp-rod internal fixation system tested on a gap model construct. Vet. Comp. Orthop. Traumatol., v.21, p.185-194, 2008.

ZAHN, K.; MATIS, U. The clamp rod internal fixator - application and results in 120 small animal fracture patients. Vet. Comp. Orthop. Traumatol., v.17, p.110120, 2004. 